\title{
General Psychiatry Clinical characteristics of hospitalised patients with schizophrenia who were suspected to have coronavirus disease (COVID-19) in Hubei Province, China
}

\author{
Xuebing Liu, ${ }^{1}$ Hong Lin, ${ }^{2}$ Haifeng Jiang, ${ }^{3}$ Ruihua Li, ${ }^{3}$ Na Zhong, ${ }^{3}$ Hang Su, ${ }^{3}$ Yi Li, ${ }^{1}$ \\ Min Zhao ${ }^{3}$
}

To cite: Liu X, Lin H, Jiang $\mathrm{H}$, et al. Clinical characteristics of hospitalised patients with schizophrenia who were suspected to have coronavirus disease (COVID-19) in Hubei Province, China. General Psychiatry 2020;33:e100222. doi:10.1136/ gpsych-2020-100222

$\mathrm{XL}$ and $\mathrm{HL}$ contributed equally.

$\mathrm{XL}$ and $\mathrm{HL}$ are joint first authors.

Received 16 March 2020

Revised 31 March 2020

Accepted 02 April 2020

\section{Check for updates}

(c) Author(s) (or their employer(s)) 2020. Re-use permitted under CC BY-NC. No commercial re-use. See rights and permissions. Published by BMJ.

${ }^{1}$ Wuhan Mental Health Center, Wuhan, China

${ }^{2}$ Yichang Special Care Hospital, Yichang, China

${ }^{3}$ Shanghai Jiao Tong University School of Medicine Affiliated Shanghai Mental Health Center, Shanghai, China

Correspondence to

Dr Min Zhao;

drminzhao@gmail.com

Ruihua Li; liruihua37@163.com

\section{ABSTRACT}

Background Since the outbreak of COVID-19, no data have been available for hospitalised psychiatric patients who are suspected to have COVID-19. We performed a comprehensive investigation of the clinical features of hospitalised patients with schizophrenia with or without suspected COVID-19 in Hubei Province, China.

Aim To explore the clinical characteristics of hospitalised patients with schizophrenia with suspected COVID-19 in Hubei Province, China.

Methods 21 hospitalised patients with schizophrenia with suspected COVID-19 (COVID-19 suspected group) in the isolation ward of a mental health hospital in Wuhan and 30 hospitalised patients with schizophrenia (clean group) in the general ward of another mental health hospital in Yichang were recruited. We retrospectively reviewed their clinical characteristics, laboratory findings and chest CT results before 21 February 2020. We also compared the emotional and mental symptoms between the two groups.

Results Medical records revealed that 21 COVID-19 suspected patients were transferred to the isolation ward between 30 January 2020 and 15 February 2020. The mean age (SD) of COVID-19 suspected patients was 43.1 (2.6). 12 (57.1\%) patients showed abnormalities on chest CT before onset of respiratory symptoms. 14 (66.7\%) patients had psychiatric medications adjustment after detection of abnormal chest CT findings. By 21 February, one patient was confirmed to have COVID-19. Even though the remaining $20(95.2 \%)$ were negative for at least two reverse transcription PCR tests, 11 (52.4\%) patients met the diagnostic criteria for clinically confirmed cases. Compared with patients in the clean group, patients in the suspected COVID-19 group showed significantly higher stress, depression and anxiety levels and poorer sleep quality.

Conclusion Setting up an independent isolation ward for hospitalised psychiatric patients who are suspected to have symptoms of COVID-19 helped control the spread of the epidemic. Patients with schizophrenia suspected to have COVID-19 showed increased stress and mood and sleep disturbances, which should be appropriately managed.

\section{INTRODUCTION}

Since the outbreak of COVID-19 caused by severe acute respiratory syndrome coronavirus 2 (SARS-CoV-2) in Wuhan, Hubei Province, China, patients with severe mental illness have inevitably been affected. Considering the understanding of COVID-19 to date, enormous challenges exist in the management of hospitalised patients with mental disorders. China has issued a series of measures, including triage, infection prevention and control, and treatment, to deal with the COVID-19 epidemic in mental health hospitals. ${ }^{12}$ Hospitalised patients with mental disorders have emotional disturbances, behavioural disorders and and a history of long-term use of antipsychotic drugs. Physical and psychiatric symptoms of patients with mental disorders may worsen with the SARS-CoV-2 infection, leading to difficulties in treatment and management. We have found no published studies on the clinical features of hospitalised psychiatric patients who have COVID-19. With these challenges in mind, we performed a comprehensive retrospective investigation of the clinical features of 21 hospitalised patients with schizophrenia who were suspected to have COVID-19 at a mental health hospital in Hubei Province, China. Further, we compared them with 30 clean hospitalised patients with schizophrenia at another mental health hospital in Hubei Province in order to better understand how COVID-19 influenced clinical outcomes.

\section{METHODS}

Study design and participants

This was a retrospective, double-centre study. Owing to the outbreak of COVID19 , isolation wards were established on 30 January 2020 at one mental health hospital 


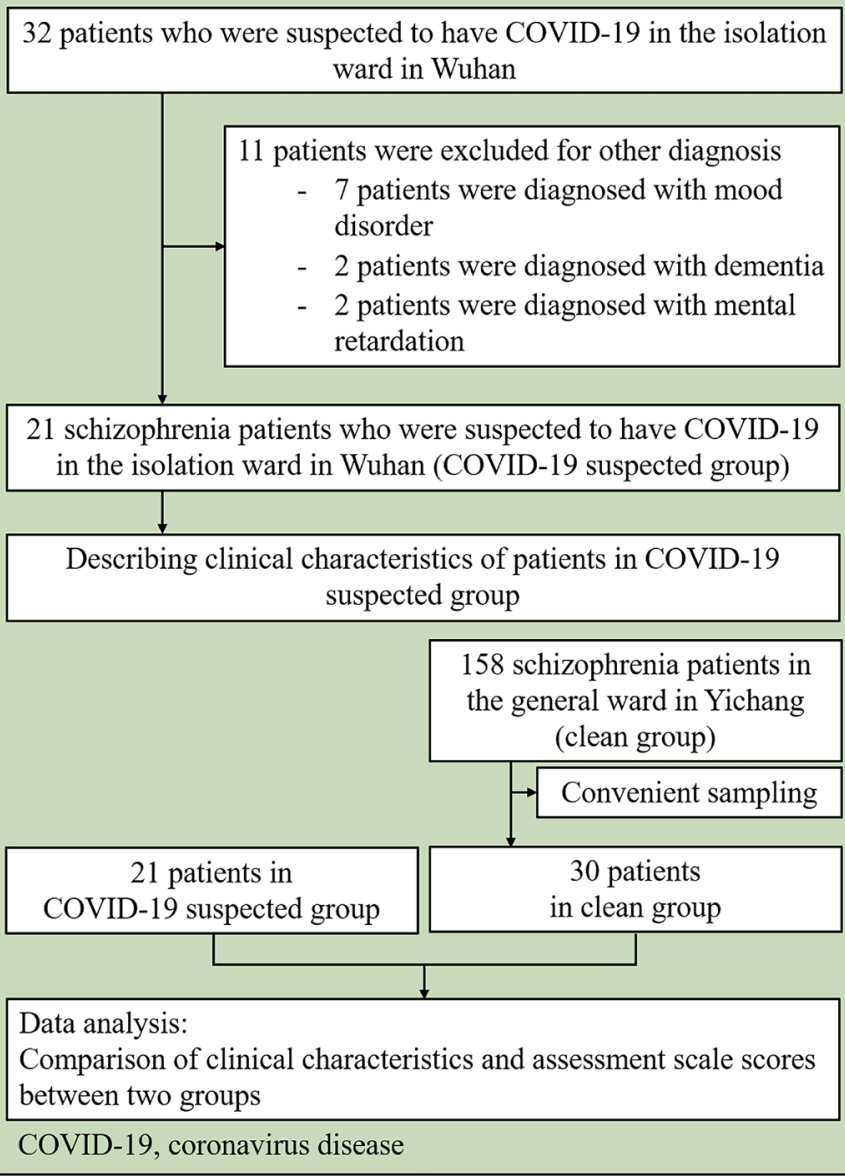

Figure 1 Flowchart of sample recruitment.

in Hubei Province. When patients in this mental health hospital showed the onset of respiratory symptoms, such as fever and cough and/or new abnormal chest CT findings, they were transferred to the isolation wards as COVID-19 suspected cases. Hospitalised patients with schizophrenia who were suspected to have COVID-19 (COVID-19 suspected group) were recruited from one of the isolation wards at this mental health hospital from 30 January to 21 February 2020. Meanwhile 30 hospitalised patients with schizophrenia in the general ward who were not suspected to have COVID-19 (clean group) from a mental health hospital in Yichang (also in Hubei Province, $300 \mathrm{~km}$ away from Wuhan) were recruited using a convenience sampling method. The two aforementioned hospitals are large psychiatric hospitals in Hubei Province, providing outpatient and inpatient treatment for patients with severe mental illness. Most wards are of the closed type, and inpatients receive medication, psychological rehabilitation and physical therapy (figure 1).

\section{Procedures}

We extracted data on clinical characteristics, radiological characteristics, laboratory findings and treatment up to 21 February 2020 from the electronic medical records of patients with suspected COVID-19. For patients in the clean group, we obtained data on clinical characteristics and treatment of mental illnesses from the electronic medical records. When the electronic medical records were unclear, we enquired with the doctors of the wards. The investigation was implemented without any intervention in the treatment of patients. After two doctors performed and verified the mental examinations, several scales were used to assess the emotional and mental symptoms of both groups. The Positive and Negative Syndrome Scale (PANSS) ${ }^{3}$ was used to assess the psychopathology associated with schizophrenia. The Perceived Stress Scale (PSS) ${ }^{4}$ was used to assess the perception of stress. The Hamilton Anxiety Rating Scale (HAMA) ${ }^{5}$ and the Hamilton Depression Rating Scale (HAMD) ${ }^{6}$ were used to assess the severity of emotional symptoms. The Pittsburgh Sleep Quality Index (PSQI) ${ }^{7}$ was used to assess the quality of sleep.

\section{Outcomes}

In this study, the following basic clinical characteristics were investigated:

- Demographic data (age and sex).

- Comorbidities (cardiovascular, digestive, endocrine and respiratory diseases).

- Respiratory symptoms (fever, cough, shortness of breath, muscle ache, headache, sore throat, rhinorrhoea, chest pain, diarrhoea, and nausea and vomiting; recorded as 'no symptom', 'one symptom' or 'more than one symptom').

The following radiological characteristics were investigated:

- Since some patients only had abnormal results on chest CT without respiratory symptoms and those with emergent respiratory symptoms underwent chest CT examination immediately, we extracted the date when there were abnormalities on chest CT for the first time in COVID-19 suspected patients.

- Whether the patients had abnormalities on chest CT before the onset of respiratory symptoms, after the onset of respiratory symptoms or without respiratory symptoms.

- Distribution of abnormalities on chest CT: recorded as 'unilateral' or 'bilateral'.

The following laboratory findings were investigated:

- Results of the real-time reverse transcription PCR (RTPCR) tests for SARS-CoV-2.

- Counts and percentages of lymphocytopaenia (lymphocytopaenia was defined as a lymphocyte count of less than 1500 cells $/ \mathrm{mm}^{3}$ ).

- Counts and percentages of leucopenia (leucopenia was defined as a lymphocyte count of less than 4000 cells $/ \mathrm{mm}^{3}$ ).

Treatment was investigated as follows:

- Counts and percentages of antibiotic and antiviral therapy.

- Counts and percentages of intravenous antibiotic therapy.

- Categories of psychiatric medications (antidepressants, benzodiazepines and mood stabilisers). 
- Adjustment of psychiatric medications after detection of abnormalities on chest CT (increase or decrease of the types or doses of psychiatric medications).

PANSS, PSS, HAMD, HAMA and PSQI scores were used to assess patients' emotional and mental symptoms and sleep quality.

For the COVID-19 suspected group, we obtained the aforementioned data. For the clean group, demographic data, comorbidities, categories of psychiatric medications, and PANSS, PSS, HAMD, HAMA and PSQI scores were obtained with the same method as those for the COVID-19 suspected group.

\section{Statistical analysis}

Continuous measurements were expressed as mean and $\mathrm{SD}$, and categorical variables as counts and percentages. Group differences in characteristics were assessed with $\chi^{2}$ statistics for categorical variables, t-test for continuous variables with a normal distribution and non-parametric test for variables without a normal distribution. Analyses of covariance (ANCOVA) and non-parametric ANCOVA were used to adjust for benzodiazepines use between the two groups for the results of PANSS, PSS, HAMD, HAMA and PSQI. The significance level for all statistical tests was set at $\mathrm{p}<0.05$ (two-tailed). We used SPSS V.22.0 and R software for data analysis.

\section{RESULTS}

Clinical characteristics of the COVIDCovid-19 suspected group There were 21 patients in the COVID-19 suspected group and 30 patients in the clean group (figure 1). Twenty-one patients in the COVID-19 suspected group in Wuhan were transferred to the isolation ward between 30 January and 15 February 2020 due to respiratory symptoms or abnormal CT findings (figure 2). Chest CT abnormalities were first detected between 12 January and 8

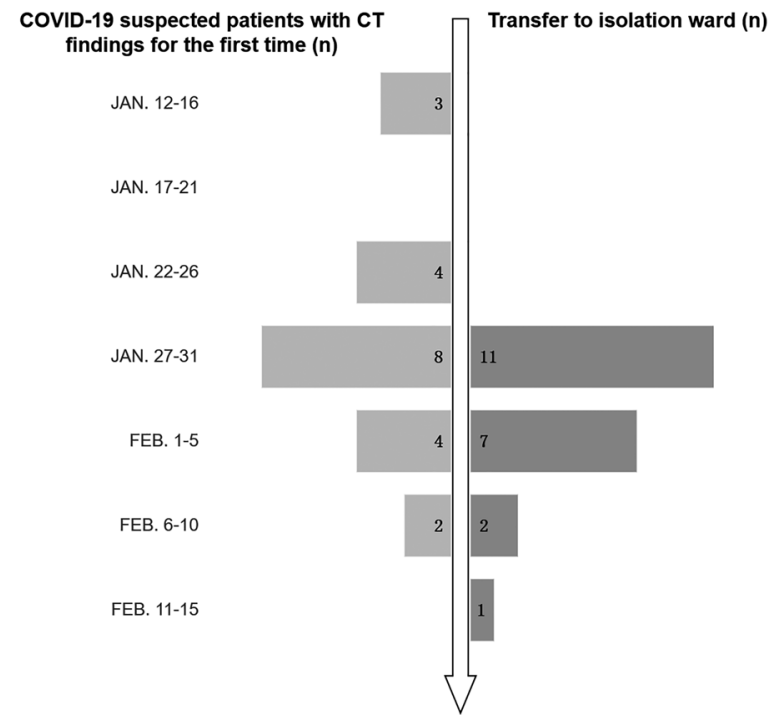

Figure 2 Flowchart of patients with COVID-19 who were transferred to the isolation ward on detection of chest CT findings for the first time.
Table 1 Clinical characteristics of patients suspected to have COVID-19

\begin{tabular}{|c|c|}
\hline & Patients $(n=21)$ \\
\hline \multicolumn{2}{|l|}{ Age (years) } \\
\hline Mean (SD) & $43.1(2.6)$ \\
\hline Range & $24-61$ \\
\hline \multicolumn{2}{|l|}{ Sex, n (\%) } \\
\hline Female & $12(57.1)$ \\
\hline Male & $9(42.9)$ \\
\hline Comorbidities, n (\%) & $8(38.1)$ \\
\hline Cardiovascular diseases & $3(14.3)$ \\
\hline Digestive disease & $4(19.0)$ \\
\hline Endocrine disease & $1(4.8)$ \\
\hline Respiratory disease & $1(4.8)$ \\
\hline \multicolumn{2}{|l|}{ Respiratory symptoms, n (\%) } \\
\hline No symptom & $5(23.8)$ \\
\hline One symptom & $8(38.1)$ \\
\hline More than one symptom & $8(38.1)$ \\
\hline \multicolumn{2}{|l|}{ Identifying the patients, n (\%) } \\
\hline $\begin{array}{l}\text { Abnormalities on chest CT before onset } \\
\text { of respiratory symptoms }\end{array}$ & $12(57.1)$ \\
\hline $\begin{array}{l}\text { Abnormalities on chest CT after onset } \\
\text { of respiratory symptoms }\end{array}$ & $4(19.0)$ \\
\hline $\begin{array}{l}\text { Abnormalities on chest CT without } \\
\text { respiratory symptoms }\end{array}$ & $5(23.8)$ \\
\hline \multicolumn{2}{|l|}{ Abnormalities on chest CT, $\mathrm{n}(\%)$} \\
\hline Unilateral & $10(47.6)$ \\
\hline Bilateral & $11(52.4)$ \\
\hline \multicolumn{2}{|l|}{ RT-PCR test, n (\%) } \\
\hline Positive & $1(4.8)$ \\
\hline Negative & $20(95.2)$ \\
\hline \multicolumn{2}{|l|}{ Routine blood test, n (\%) } \\
\hline Leucopenia & $11(52.4)$ \\
\hline Lymphocytopaenia & $9(42.9)$ \\
\hline \multicolumn{2}{|l|}{ Respiratory treatment, n (\%) } \\
\hline Antiviral treatment & $21(100)$ \\
\hline Antibiotic treatment & $21(100)$ \\
\hline Intravenous antibiotic therapy & $4(19.0)$ \\
\hline $\begin{array}{l}\text { Psychiatric treatment adjustment after } \\
\text { detection of chest CT findings, } n(\%)\end{array}$ & $14(66.7)$ \\
\hline Increase of the types or doses & $11(52.4)$ \\
\hline Increased benzodiazepines & $6(28.6)$ \\
\hline Decrease of the types or doses & $3(14.3)$ \\
\hline
\end{tabular}

RT-PCR, reverse transcription PCR.

February 2020, and eight (38.1\%) patients first showed abnormal chest CT findings between 27 January and 30 January 2020 (figure 2). Table 1 summarises the clinical characteristics of the COVID-19 suspected group. The COVID-19 suspected group included 12 (57.1\%) women 
Table 2 Comparison of clinical characteristics and assessment scale scores between patients with and without suspected COVID-19

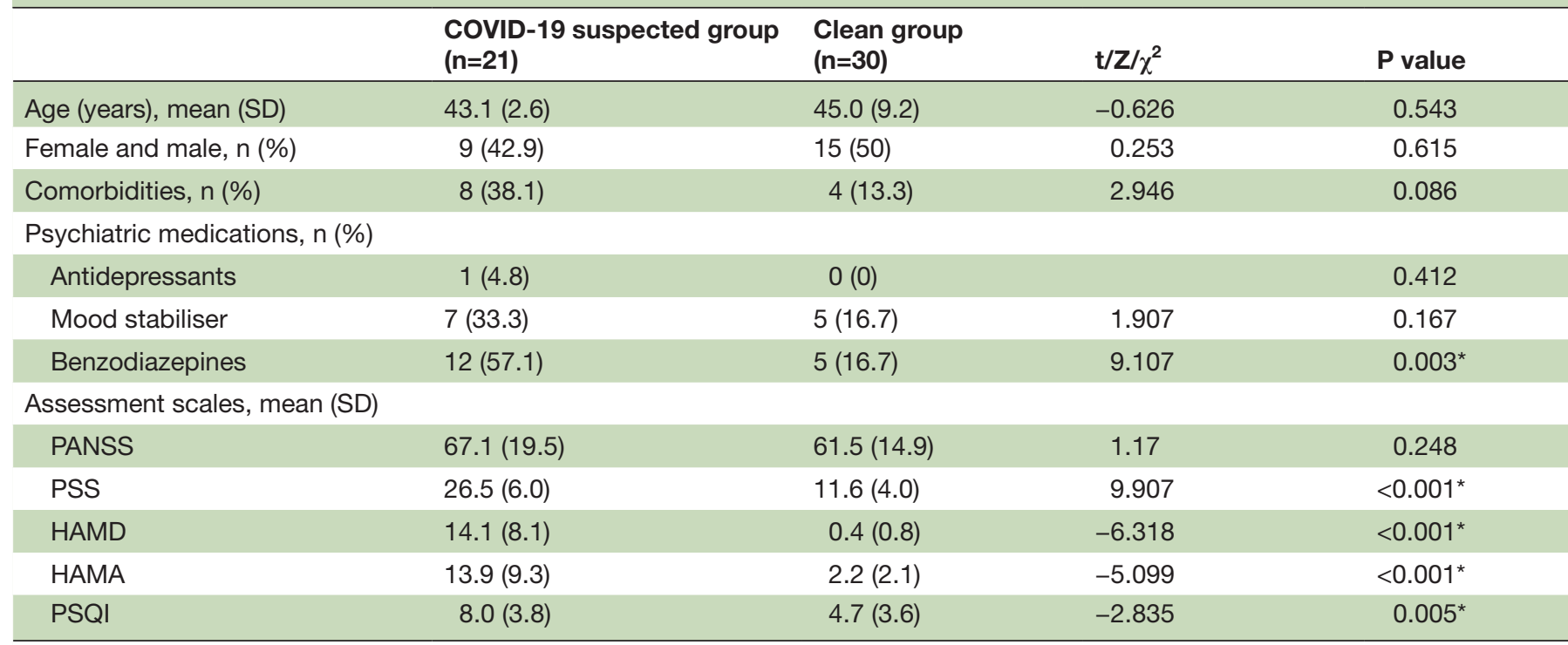

${ }^{*} \mathrm{P}<0.05$

HAMA, Hamilton Anxiety Rating Scale; HAMD, Hamilton Depression Rating Scale; PANSS, Positive and Negative Syndrome Scale; PSQI, Pittsburgh Sleep Quality Index; PSS, Perceived Stress Scale.

and $9(42.9 \%)$ men, with a mean (sd) age of $43.1(2.6)$ years. Among them, eight $(38.1 \%)$ patients had comorbidities. Eight $(38.1 \%)$ patients had more than one respiratory symptom. Twelve $(57.1 \%)$ patients had abnormalities on chest CT before onset of respiratory symptoms; four $(19.0 \%)$ had abnormalities on chest CT after onset of respiratory symptoms; and five $(23.8 \%)$ only had abnormalities on chest CT without respiratory symptoms. Eleven $(52.4 \%)$ patients had bilateral abnormalities on chest CT. Eleven (52.4\%) patients had lymphocytopaenia and nine $(42.9 \%)$ had leucopenia.

All patients underwent the RT-PCR test for SARS-CoV-2 at least twice. One $(4.8 \%)$ patient was confirmed to have COVID-19 on the third RT-PCR test. The remaining 20 (95.2\%) patients were negative for at least two PCR tests, and $11(52.4 \%)$ patients met the diagnostic criteria for clinically confirmed cases. The patient confirmed to have COVID-19 was transferred to a ward for confirmed cases on 21 February 2020.

\section{Treatment in the COVID-19 suspected group}

Patients in the COVID-19 suspected group were treated in the isolation ward which had 32 patients with 7 doctors and 14 nurses. Standard precautions were adopted in the ward. All patients received antiviral and antibiotic treatments, and four $(19.0 \%)$ received intravenous antibiotic therapy. As for psychiatric treatment, 14 (66.7\%) patients had psychiatric medication adjustment after detection of chest CT abnormalities; increase in the types or doses of psychiatric medications were found in 11 $(52.4 \%)$ patients, and $6(28.6 \%)$ patients were prescribed with an increased dose of benzodiazepines. Apart from psychiatric medications, patients received education on
COVID-19, psychological intervention and assistance for communication with their family members.

\section{Comparison between COVID-19 suspected and clean groups}

Patients in the clean group were treated in the general ward of another mental health hospital in Yichang. In addition to the daily treatment, this hospital adopted measures such as suspending family visits and conducting daily temperature tests on medical staff and hospitalised patients. There were no hospitalised patients with confirmed or suspected COVID-19 in this hospital by 21 February 2020.

The clean group included $15(50 \%)$ women and 15 $(50 \%)$ men, with a mean (SD) age of 45.0 (9.2) years. Four $(13.3 \%)$ patients had comorbidities. There were no significant differences in age, sex or percentage of comorbidities between the two groups. More patients in the COVID-19 suspected group received benzodiazepines $(12,57.1 \%)$ than in the clean group $(5,16.7 \%)$, and the difference was statistically significant $(\mathrm{p}=0.003)$. Compared with patients in the clean group, patients in the COVID-19 suspected group had significantly higher PSS, HAMD, HAMA and PSQI scores. PANSS scores showed no statistically significant difference between the two groups (table 2). After adjustment for benzodiazepine use, statistically significant difference in PSS $(\mathrm{F}=89.83, \mathrm{p}<0.001)$, HAMD $(p=0.003)$, HAMA $(p=0.003)$ and PSQI $(p=0.04)$ scores between the two groups still existed (table 3 ).

\section{DISCUSSION}

\section{Main findings}

In this retrospective study, we described the clinical characteristics of hospitalised patients with schizophrenia 
Table 3 Comparison of clinical characteristics and assessment scale scores between patients with and without suspected COVID-19 adjusting for benzodiazepine use

\begin{tabular}{llcc}
\hline & Statistical method & F & P value \\
\hline PANSS & ANCOVA & 0.547 & 0.463 \\
PSS & ANCOVA & 89.83 & $<0.001^{*}$ \\
HAMD & Non-parametric ANCOVA & - & $0.003^{*}$ \\
HAMA & Non-parametric ANCOVA & - & $0.003^{*}$ \\
PSQI & Non-parametric ANCOVA & - & $0.04^{*}$ \\
\hline
\end{tabular}

${ }^{*} \mathrm{P}<0.05$.

ANCOVA, analysis of covariance; HAMA, Hamilton Anxiety Rating Scale; HAMD, Hamilton Depression Rating Scale; PANSS, Positive and Negative Syndrome Scale; PSQI, Pittsburgh Sleep Quality Index; PSS, Perceived Stress Scale.

who were suspected to have COVID-19 in Hubei Province, China, and provided an overview of the COVID-19 suspected patients in the isolation ward of a mental health hospital. These were compared with patients with schizophrenia in the general ward of another mental health hospital to show the emotional and behavioural characteristics of patients with suspected COVID-19.

Since the outbreak of COVID-19 in Hubei Province, China, infection of SARS-CoV-2 has occurred at mental health hospitals due to contact with patients with confirmed COVID-19 or carriers during the early phase of the epidemic. We believed it was difficult to identify confirmed cases from a large number of suspected cases in the early phase of the outbreak. Identifying COVID-19 infection based on clinical symptoms and chest CT, rather than relying entirely on RT-PCR testing, helped patients receive timely treatment and reduced the risk of exposure among uninfected patients. In this study, only one patient had positive result on the third RT-PCR test, and although the remaining 20 patients were negative for at least two PCR tests, $11(52.4 \%)$ met the diagnostic criteria for clinically confirmed cases. False-positive results of the RT-PCR test for SARS-CoV-2 have been reported due to clinical course, specimen collection and detection process, ${ }^{8}$ through which some patients may be underdiagnosed. In this study, some patients had abnormalities on chest CT without respiratory symptoms, and more than half of patients had chest CT findings before the onset of respiratory symptoms. Recent studies have reported patients with chest CT findings who initially had negative results in the RT-PCR test for SARS-CoV-2 and finally positive results. ${ }^{910}$ The asymptomatic patients with COVID-19 were not well understood and such cases bring challenges to infection control, ${ }^{11}{ }^{12}$ particularly in places with closed management such as mental health hospitals. Therefore, according to the fifth edition of the guideline on COVID-19 in China (valid from 5-19 February), ${ }^{13} 14$ timely centralised isolation of patients with suspected symptoms at that time had positive significance in controlling the spread of the epidemic at the mental health hospital.
As for psychiatric management, psychiatric medications were adjusted according to the clinical progress of patients with suspected COVID-19. More than half of the patients received increased prescriptions of the types or doses. Meanwhile, psychotherapy and patient education were strengthened. Both respiratory treatment and original psychiatric management should be taken into account for hospitalised patients with schizophrenia who are suspected to have COVID-19. ${ }^{2}$ Strengthening the assessment of psychiatric symptoms and monitoring biochemical levels were suggested in future treatment. ${ }^{2}$

Greater perceived stress and emotions of depression and anxiety were found in the COVID-19 suspected group than in the clean group. Moreover, patients in the COVID-19 suspected group had poorer sleep quality. Transferring to the isolation ward, changes in environment and healthcare workers, and suspected COVID-19 itself could be stressors for patients. ${ }^{15}$ Depression, anxiety and poor sleep quality could occur under stress. ${ }^{16-18}$ The lessons learnt from the severe acute respiratory syndrome and Ebola outbreaks indicated that challenges and stress could trigger common mental disorders, including anxiety and depressive disorders, and post-traumatic stress disorder, ${ }^{19}{ }^{20}$ which in turn could result in hazards that exceed the consequences of the infection itself. ${ }^{21}$ Besides, ACE2, the receptor of SARS-CoV-2, ${ }^{22}$ is a component of the renin-angiotensin system (RAS) which can be found in the central nervous system. ${ }^{23-25}$ Animal studies indicated that RAS is related to stress and mood disorders. ${ }^{24}{ }^{25}$ Whether SARS-CoV-2 could infect the central nervous system and cause affect stress and emotional disturbances or not needs to be investigated. Comprehensive clinical measures should be taken in the isolation ward to help patients cope with these problems.

\section{Limitations}

There were several limitations to this study. First, this was a retrospective study focusing on the clinical characteristics and management of hospitalised patients with schizophrenia who were suspected to have COVID-19, with statistical analysis that was relatively simple. Longitudinal studies focusing on patients with schizophrenia who have COVID-19 are required in the future. Second, patients in the COVID-19 suspected group and clean group were recruited from different hospitals and the assessments were performed by different doctors, which may cause bias. In order to reduce bias, we selected two mental health hospitals with similar scales in Wuhan, and the doctors who performed the assessment of patients were at least attending doctors. Third, follow-up of patients in the COVID-19 suspected group is needed to reveal more details with regard to clinical characteristics.

\section{Implications}

This study depicted the clinical features of hospitalised patients with schizophrenia who were suspected to have COVID-19. The process of management, such as distinguishing suspected patients and transferring them to the 
isolation ward, was described in detail. This study revealed that setting up an independent isolation ward for treating psychiatric patients who were suspected to have COVID-19 helped control the spread of the epidemic at a mental health hospital. Patients with schizophrenia suspected of an infection with SARS-CoV-2 showed obvious stress and mood and sleep disturbances, which should be accounted for in the management of these patients.

Contributors XL, HL and RL collected the clinical data. RL, HS and NZ processed the statistical data. RL drafted the manuscript. $\mathrm{HJ}$ and $\mathrm{YL}$ revised the final manuscript. MZ is responsible for summarising all data and revised the final manuscript.

Funding This work was supported by the Program of Shanghai Academic Research Leader (17XD1403300) and the Shanghai Key Laboratory of Psychotic Disorders (13DZ2260500)

Disclaimer The funders have no role in study design, data collection and analysis, and decision to publish or preparation of the manuscript.

Competing interests None declared.

Patient consent for publication Not required.

Ethics approval This study was approved by the Ethics Committee of the Shanghai Mental Health Center (no 2019-18) and was carried out in accordance with the principles of the Declaration of Helsinki. Written informed consent was obtained from patients involved or their guardians.

Provenance and peer review Not commissioned; externally peer reviewed.

Data availability statement Data are available upon reasonable request.

Open access This is an open access article distributed in accordance with the Creative Commons Attribution Non Commercial (CC BY-NC 4.0) license, which permits others to distribute, remix, adapt, build upon this work non-commercially, and license their derivative works on different terms, provided the original work is properly cited, appropriate credit is given, any changes made indicated, and the use is non-commercial. See: http://creativecommons.org/licenses/by-nc/4.0/.

\section{REFERENCES}

1 Chinese Society of Psychiatry, Psychiatric Hospital Branch of Chinese Hospital Association, Chinese Preventive Medicine Association, Society of Mental Health, Chinese Psychiatrist Association, Chinese Society of Neuroscience \& Psychiatry. Expert consensus on managing pathway and coping strategies for patients with mental disorders during prevention and control of serious and outbreak infectious diseases (novel coronavirus pneumonia). Chin J Psychiatry 2020;53.

2 Chinese Society of Psychiatry, Chinese Psychiatrist Association. Psychopharmacology therapy recommendation for patients with COVID-19. Chin J Psychiatry 2020;53.

3 Kay SR, Opler LA, Lindenmayer JP. The positive and negative syndrome scale (PANSS): rationale and standardisation. $\mathrm{Br} \mathrm{J}$ Psychiatry Suppl 1989;7:59-65.

4 Cohen S, Kamarck T, Mermelstein R. A global measure of perceived stress. J Health Soc Behav 1983;24:385-96.

5 HAMILTON M. The assessment of anxiety states by rating. Br J Med Psychol 1959;32:50-5.
6 HAMILTON M. A rating scale for depression. $J$ Neurol Neurosurg Psychiatry 1960;23:56-62.

7 Buysse DJ. Reynolds CF 3rd, monk th, Berman SR, Kupfer DJ. The Pittsburgh sleep quality index: a new instrument for psychiatric practice and research. Psychiatry Res 1989;28:193-213.

8 Li Jin YG, Liangjun C, et al. Analysis of false-negative results for 2019 novel coronavirus nucleic acid test and related countermeasures. Chin J Lab Med 2020;43.

9 Huang P, Liu T, Huang L, et al. Use of chest CT in combination with negative RT-PCR assay for the 2019 novel coronavirus but high clinical suspicion. Radiology 2020;295:22-3.

10 Xie X, Zhong Z, Zhao W, et al. Chest CT for typical 2019-nCoV pneumonia: relationship to negative RT-PCR testing. Radiology 2020;200343:200343.

11 Al-Tawfiq JA. Asymptomatic coronavirus infection: MERS-CoV and SARS-CoV-2 (COVID-19). Travel Med Infect Dis 2020;101608.

12 Pan X, Chen D, Xia Y, et al. Asymptomatic cases in a family cluster with SARS-CoV-2 infection. Lancet Infect Dis 2020;S14733099:30114-6.

13 General Office of National Health Committee. Office of state administration of traditional Chinese medicine. notice on the issuance of a programme for the diagnosis and treatment of novel coronavirus (2019-nCoV) infected pneumonia (trial version 5), 2020. Available: http://www.nhc.gov.cn/yzygj/s7653p/202002/ 3b09b894ac9b4204a79db5b8912d4440.shtml [Accessed 7 Mar 7 2020].

14 General Office of National Health Committee. Office of state administration of traditional Chinese medicine. notice on the issuance of a programme for the diagnosis and treatment of novel coronavirus (2019-nCoV) infected pneumonia (trial version 5, revised version), 2020. Available: http://www.nhc.gov.cn/yzygj/s7653p/ 202002/d4b895337e19445f8d728fcaf1e3e13a.shtml [Accessed 7 Mar 2020].

15 Godoy LD, Rossignoli MT, Delfino-Pereira P, et al. A comprehensive overview on stress neurobiology: basic concepts and clinical implications. Front Behav Neurosci 2018;12:127.

16 Yang L, Zhao Y, Wang Y, et al. The effects of psychological stress on depression. Curr Neuropharmacol 2015;13:494-504.

17 Herbison CE, Allen K, Robinson M, et al. The impact of life stress on adult depression and anxiety is dependent on gender and timing of exposure. Dev Psychopathol 2017;29:1443-54.

18 Kalmbach DA, Anderson JR, Drake CL. The impact of stress on sleep: pathogenic sleep reactivity as a vulnerability to insomnia and circadian disorders. J Sleep Res 2018;27:e12710.

19 Mak IWC, Chu CM, Pan PC, et al. Long-term psychiatric morbidities among SARS survivors. Gen Hosp Psychiatry 2009;31:318-26.

20 Shultz JM, Baingana F, Neria Y. The 2014 Ebola outbreak and mental health: current status and recommended response. JAMA 2015;313:567-8.

21 Qiu J, Shen B, Zhao M, et al. A nationwide survey of psychological distress among Chinese people in the COVID-19 epidemic: implications and policy recommendations. Gen Psychiatr 2020;33:e100213.

22 Zhou P, Yang X-L, Wang X-G, et al. A pneumonia outbreak associated with a new coronavirus of probable bat origin. Nature 2020:579:270-3.

23 Hamming I, Timens W, Bulthuis MLC, et al. Tissue distribution of ACE2 protein, the functional receptor for SARS coronavirus. A first step in understanding SARS pathogenesis. J Pathol 2004;203:631-7.

24 Rocha NP, Simoes E Silva AC, Prestes TRR, et al. RAS in the central nervous system: potential role in neuropsychiatric disorders. Curr Med Chem 2018;25:3333-52.

25 Jackson L, Eldahshan W, Fagan S, et al. Within the brain: the renin angiotensin system. Int J Mol Sci 2018;19:876.

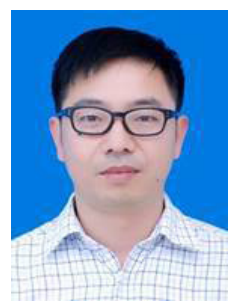

Dr Xuebing Liu obtained a doctorate from Central South University Xiangya School of Medicine and now work as the director of addiction center of Wuhan mental health center. He is the member of Chinese Association of Drug Abuse Prevention and Treatment, Addiction Medicine Group of Chinese Society of Psychosomatic Medicine, and Committee of Drug Dependence $\mathcal{E}$ Abuse of Chinese Society of Toxicology, and the secretary of Chinese Synthetic Drug Association of Chinese Association of Drug Abuse Prevention and Treatment. He also participates in many other national and local academic committees. His major research interests include psychiatry and mechanisms, prevention and treatment of addiction. 


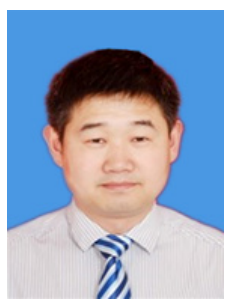

Dr Hong Lin, deputy director and psychotherapist. He obtained the master's degree from Wuhan University, and now he worked as the chief of Medical Department and Department of Medical Records in Yichang Special Care Hospital, and director of Teaching office of mental health center in China Three Gorges University. He was the member of Chinese Psychiatrist Association and Chinese Association of Drug Abuse Prevention and Treatment. He also participates in many other local academic committees. Besides, he takes in charge or participates in many national, provincial and municipal scientific research projects. 